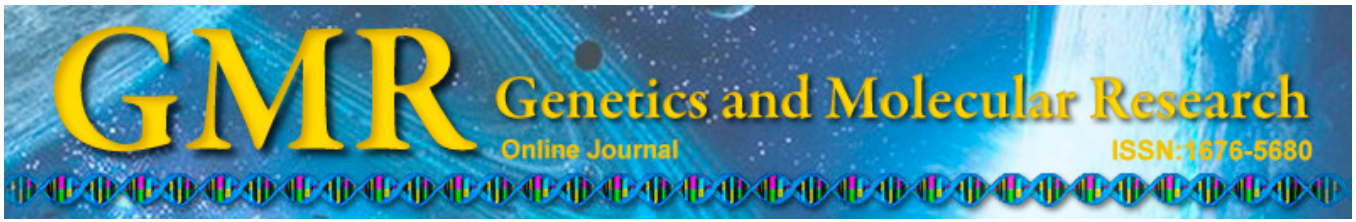

\title{
Effects of the Zhikang capsule on healing of the flap after radical breast cancer surgery
}

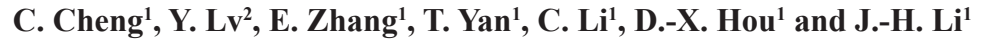 \\ ${ }^{1}$ Department of Surgical Oncology, \\ The Third Affiliated Hospital of the Medical College of Xi' an Jiaotong \\ University (Shaanxi Provincial People's Hospital), Shaanxi, Xi'an, China \\ ${ }^{2}$ Department of Hepatobiliary Surgery, \\ The First Affiliated Hospital of the Medical College of Xi' an Jiaotong University, \\ Shaanxi, Xi'an, China \\ Corresponding authors: Y. Lv / J.-H. Li \\ E-mail: luyi169@126.com / lihuijian_1hj@163.com
}

Genet. Mol. Res. 14 (2): 5127-5131 (2015)

Received September 15, 2014

Accepted December 11, 2014

Published May 18, 2015

DOI http://dx.doi.org/10.4238/2015.May.18.2

\begin{abstract}
This study aimed to investigate the effect and mechanism of trauma flap healing promoted by the Zhikang capsule after radical breast cancer surgery. The enrolled breast cancer patients were randomly divided into two groups: treatment and observation. The patients in the treatment group were treated with the Zhikang capsule in addition to the conventional dressing changes, while patients in the observation group underwent only the regular dressing changes. Serum samples of 98 breast cancer patients (with complete clinical data) who underwent modified radical mastectomy were collected and analyzed for expressions of transforming growth factor beta (TGF- $\beta$ ) and basic fibroblast growth factor (bFGF). The drainage fluid amount and tissue necrosis rate were found to be lower in the treatment group than in the observation group. Moreover, bFGF expression in peripheral blood was higher in the treatment group than in the observation group. However, no significant difference was found between the two groups in the expression of TGF- $\beta$ in peripheral blood. In conclusion, Zhikang
\end{abstract}


capsule is effective in promoting flap healing after radical breast cancer surgery, and the increase of bFGF expression in peripheral blood may be the underlying mechanism.

Key words: Zhikang capsule; Breast cancer; Flap

\section{INTRODUCTION}

Flap necrosis, infection, and subcutaneous fluid commonly occur after modified radical mastectomy. Delayed wound healing caused by flap necrosis leads to not only unnecessary suffering in patients, but also prolonged hospitalization and increased economic burden, as well as delay in the implementation of other therapeutic regimens. For these reasons, surgeons have searched for effective methods to promote wound healing. In the present study, we selected 98 patients who underwent modified radical mastectomy. We examined their state of subcutaneous fluid volume, extubation time, and flap necrosis, and their serum transforming growth factor beta (TGF- $\beta$ ) and basic fibroblast growth factor (bFGF) expression levels by immunohistochemistry after the application of Zhikang capsule, with the aim to investigate its effect on flap healing after radical breast cancer surgery and the variations in the expression of wound healing related cytokines.

\section{MATERIAL AND METHODS}

\section{Materials}

Ninety-eight women who underwent modified radical mastectomy in our hospital between January 2011 and March 2012 were enrolled in this study. They were randomly divided into two groups, each with 49 patients. The patients in the observation group (median age, 42 years; age range, 33-72 years) received only conventional dressing treatment, and those in the treatment group (median age, 44 years; age range, 29-74 years) also received the Zhikang capsule (Xi'an Qianhe Pharmaceutical Co.) for 14 days from the first day after surgery, at a dosage of 0.3 t.i.d. There were no significant differences in age, gender, tumor size, and surgical method between the two groups.

\section{Postoperative status}

Wound healing was observed and recorded on postoperative day $3,7,10$, and 14 . Subcutaneous fluid volume, extubation time, flap necrosis, and flap healing time were recorded and compared between the two groups.

\section{Cytokine detection}

The expression levels of serum TGF- $\beta$ and bFGF were measured by enzyme-linked immunosorbent assay (ELISA) 1 day before the surgery and again on the 7 th and 14th day after surgery. The relationship between wound healing and the changes in these two indicators were analyzed. ELISA kits for TGF- $\beta$ and bFGF detection were purchased from R \& D companies (USA). 


\section{Statistical analysis}

Data were processed with the SPSS 15.0 software, and reported as means \pm standard deviation. The groups were compared using the $t$-test.

\section{RESULTS}

\section{Wound status}

We found that the number of necrotic cases in the observation group was significantly higher than that in the treatment group $(\mathrm{P}<0.05)$; the drainage fluid amount in the treatment group $(273.1 \pm 233.03 \mathrm{~mL})$ was significantly lower than that in the observation group (662.2 $\pm 309.28 \mathrm{~mL})(\mathrm{P}<0.05)$; and postoperative drainage tube removal time in the treatment group was significantly shorter than that in the observation group $(\mathrm{P}<0.05)($ Table 1$)$.

Table 1. General characteristics of trauma flap healing in two groups.
\begin{tabular}{lccc}
\hline Group & Tissue necrosis & Drainage fluid $(\mathrm{mL})$ & Drainage tube removal (day) \\
\hline Observation & 7 & $662.2 \pm 309.28$ & $11.8 \pm 6.18$ \\
Treatment & 2 & $273.1 \pm 233.03$ & $6.2 \pm 2.17$ \\
$\mathrm{P}$ & $<0.05$ & $<0.05$ & $<0.05$ \\
\hline
\end{tabular}

\section{Detection of serum cytokine}

As shown in Table 2, there were no significant differences between the two groups in the expression levels of TGF- $\beta$ one day before modified radical mastectomy, or 7 or 14 days after the surgery $(\mathrm{P}>0.05)$.

However, we found that the expression levels of bFGF on the 7th and 14th days after modified radical mastectomy were significantly higher in the patients of the treatment group than in the patients of the observation group $(\mathrm{P}<0.05)$, as shown in Table 3.

Table 2. Expression of TGF- $\beta$ in the serum of two groups.
\begin{tabular}{lccc}
\hline Group & One day prior to surgery & After surgery \\
\cline { 2 - 3 } & & 7 th day & 14 th day \\
\hline Observation & $0.1132 \pm 0.0734$ & $0.1966 \pm 0.1233$ & $0.1359 \pm 0.0499$ \\
Treatment & $>.1621 \pm 0.0359$ & $0.1545 \pm 0.1252$ & $0.1308 \pm 0.0196$ \\
$P$ & $>0.05$ & $>0.05$ & $>0.05$ \\
\hline
\end{tabular}

Table 3. Expression of bFGF in the serum of two groups.

\begin{tabular}{lccr}
\hline Group & One day prior to surgery & \multicolumn{2}{c}{ After surgery } \\
\cline { 3 - 4 } & & 7th day & 14 th day \\
\hline Observation & $0.1488 \pm 0.0337$ & $0.1399 \pm 0.0294$ & $0.1369 \pm 0.0209$ \\
Treatment & $0.9835 \pm 0.1204$ & $1.766 \pm 1.722$ & $1.599 \pm 1.5395$ \\
$\mathrm{P}$ & $<0.01$ & $<0.05$ & $<0.05$ \\
\hline
\end{tabular}




\section{DISCUSSION}

Modified radical mastectomy improves patient survival rate as well as the postoperative appearance of the patient, as it retains breast muscle. However, due to a variety of factors including preoperative incision choice, surgery, and postoperative treatment, flap necrosis and subcutaneous fluid are more commonly seen after modified radical mastectomy than after conventional mastectomy. The domestic and international incidence of flap necrosis has reached $10-60 \%$ (Kim et al., 2004). Flap necrosis causes delayed wound healing, which leads to not only unnecessary suffering in patients, but also prolonged hospitalization and increased economic burden. Furthermore, delay in implementation of other therapeutic regimens caused by the above-mentioned reasons severely impacts the prognosis of these patients. In the present study, we found that wound healing in the patients of the treatment group, who were treated with the Zhikang capsule, was significantly better than that in the observation group; thus proving the promoting effect of Zhikang capsule on wound healing, which has been previously reported by other studies (Meng and Liu, 2001).

Injury to tissues and organs of the body due to various harmful factors causes cell injury and leads to formation of defects; these defects can be repaired via regeneration of the same or different types of cells, ultimately resulting in wound closure. Numerous cytokines have been found to be involved in this process; among these, TGF- $\beta$ and bFGF are important cytokines.

TGF- $\beta$, a crucial bioactive molecule in the wound healing process, has a chemotactic effect, and promotes scar formation, alters the wound healing schedule, and controls fibrosis. Together with Bletilla striata gum, one of the effective components of the Zhikang capsule, TGF- $\beta$ plays a role in promoting wound granulation tissue growth and increasing wound exudation of polymorphonuclear leukocytes, monocytes, and fibroblasts, thereby improving tissue repair (Chai, 2003). Yet circulating TGF- $\beta$ seems to be split apart with that localized in the wound. In 1987, Mustoe et al. (1987) reported that exogenous TGF- $\beta$ could increase the tensile strength of the repaired cut wounds in male rats by $20 \%$, and accelerate wound healing by inducing smooth muscle actin synthesis of dermal cells, which could cause skin wound contraction. However, scar reduction was noted in transgenic mice that had higher TGF- $\beta$ levels in the peripheral blood, suggesting that TGF- $\beta$ in the peripheral blood was no substitute for TGF- $\beta$ in the local tissue in regard to wound healing, and that TGF- $\beta$ during wound healing was primarily from the local injured tissue rather than from the blood circulation. A complete understanding of the changes of localized TGF- $\beta$ requires further investigation. On the other hand, bFGF can induce formation, proliferation, and differentiation of microvessels (Rabie and Lu, 2004), and stimulate proliferation of all wound repair cells. In early trauma, bFGF was secreted by macrophages and endothelial cells localized in the wound to stimulate proliferation and migration of fibroblasts and keratinocytes, leading inflammatory cells and surrounding cells to the wound surface and inducing the production of protease, collagenase, and various cytokines. Use of bFGF for the treatment of refractory burn wounds in mice with diabetes, malnutrition, and other similar conditions resulted in a significant acceleration of wound contraction and closure (Okumura et al., 1996a,b; Wang and Han, 2002). In a study by Fu et al. (1999) on 33 chronic refractory wounds in 28 cases, after the treatment with exogenous bFGF, the cure rate of these refractory wounds (unhealed after 4 weeks of traditional treatment) reached $100 \%$, and the 4 week-healing rate was $90.9 \%$. Histological examination of these wounds showed that the number of capillaries and fibroblasts increased significantly in those treated with bFGF. However, the healing promoting effect of bFGF differs with wound 
types; the effect increases in the order of fresh wounds, burn wounds, and chronic wounds. Numerous experimental results have demonstrated that an application of exogenous bFGF can significantly improve the healing of chronic refractory wounds, with the premise of etiological treatment such as diabetes control and circulation improvement (Liu and Tao, 2009). Additionally, elementary surgical debridement and wound anti-infection treatment also provide the basis for wound healing when cytokines are applied. The best conditions for growth factors to promote healing of chronic refractory wounds are based on debridement and infection control. From the pharmacological action of each component in the Zhikang capsule, we may conjecture that: 1) Bletilla striata gum in Bletilla striata can promote the growth of wound granulation tissue and increase wound exudation of polymorphonuclear leukocytes, monocytes, and fibroblasts, thus contributing to tissue repair and anti-infection; and 2) Pearl powder has an antioxidant effect. The total porphyrin in pearl powder and its products can inhibit free radical reactions and promote cell proliferation and differentiation, thus improving blood circulation on the wound surface and accelerating wound healing (Meng and Liu, 2001; Wang et al., 2004). All the above effects are consistent with the wound healing promoting mechanism of bFGF. In addition, our results demonstrated a higher level of bFGF expression in the peripheral blood of patients in the treatment group, indicating that serum bFGF expression may be related to the flap healing effect of the Zhikang capsule after radical mastectomy. Further studies are needed to reveal the specific mechanisms.

\section{ACKNOWLEDGMENTS}

Research supported by the 2011 Traditional Chinese Medicine Science and Technology Research Projects of Shaanxi Province Administration of Traditional Chinese Medicine (\#JC25) and the 2012 Natural Science Basic Research Program of Shaanxi Province (\#2012JM4033).

\section{REFERENCES}

Chai SF (2003). 21 cases of upper gastrointestinal bleeding treated by decoction of Bletilla striata and rhubarb. China Foreign Med. J. 4: 78.

Fu XB, Guo ZR and Sheng ZY (1999). Effects of basic fibroblast growth factor in the healing of cutaneous chronic wounds. Chin. J. Reparative Reconstr. Surg. 13: 270-272.

Kim LS, Huang S, Lu W, Lev DC, et al. (2004). Vascular endothelial growth factor expression promotes the growth of breast cancer brain metastases in nude mice. Clin. Exp. Metastasis 21: 107-118.

Liu JY and Tao AM (2009). Clinical efficacy of Zhikang capsule in the treatment of internal hemorrhoid hemorrhage. Clin. J. Med. Officer 37: 422.

Meng QS and Liu K (2001). Clinical observation of soft tissue injury and wound infection treated by Zhikang capsule. China Pharmaceuticals 10: 55-56.

Mustoe TA, Pierce GF, Thomason A, Gramates P, et al. (1987). Accelerated healing of incisional wounds in rats induced by transforming growth factor-beta. Science 237: 1333-1336.

Okumura M, Okuda T, Nakamura T and Yajima M (1996a). Acceleration of wound healing in diabetic mice by basic fibroblast growth factor. Biol. Pharm. Bull. 19: 530-535.

Okumura M, Okuda T, Nakamura T and Yajima M (1996b). Effect of basic fibroblast growth factor on wound healing in healing impaired animal models. Arzneimittelforschung 46: 547-551.

Rabie AB and Lu M (2004). Basic fibroblast growth factor up-regulates the expression of vascular endothelial growth factor during healing of allogeneic bone graft. Arch. Oral Biol. 49: 1025-1033.

Wang Y, Zhou YS, Xiong J and Zhang JX (2004). Effects of Zhikang capsule on the hemorheology indexes in patients with acute trauma. Herald Med. 23: 156-157.

Wang ZT and Han C (2002). Observation of basic fibroblast growth factor in promoting the proportion of wound healing after anorectal surgery. Chin. New Med. 3: 686-687. 